\title{
Modular inflation observables and $j$-inflation phenomenology
}

\section{Rolf Schimmrigk}

Indiana University South Bend, 1700 Mishawaka Ave., South Bend, IN 46634, U.S.A.

E-mail: rschimmr@iusb.edu

ABSTRACT: Modular inflation is the restriction to two fields of automorphic inflation, a general group based framework for multifield scalar field theories with curved target spaces, which can be parametrized by the comoving curvature perturbation $\mathcal{R}$ and the isocurvature perturbation tensor $S^{I J}$. This paper describes the dynamics and observables of these perturbations and considers in some detail the special case of modular inflation as an extensive class of two-field inflation theories with a conformally flat target space. It is shown that the nonmodular nature of derivatives of modular forms leads to CMB observables in modular invariant inflation theories that are in general constructed from almost holomorphic modular forms. The phenomenology of the model of $j$-inflation is compared to the recent observational constraints from the PLANCK satellite and the BICEP2/Keck Array data.

KEYwords: Cosmology of Theories beyond the SM, Discrete Symmetries

ARXIV EPRINT: 1612.09559 


\section{Contents}

1 Introduction 1

2 Multifield dynamics $\quad 3$

2.1 Scalar field dynamics 3

2.2 Curved target background dynamics 4

2.3 Slow-roll dynamics 5

3 Perturbed multifield inflation $\quad 6$

3.1 Dynamics of perturbations 6

3.2 The slow-roll form of the power spectrum 8

$\begin{array}{ll}3.3 & \text { The tensor-to-scalar ratio }\end{array}$

4 Modular inflation $\quad 9$

$\begin{array}{lll}4.1 & \text { Kinetic term } & 10\end{array}$

$\begin{array}{lll}4.2 & \text { Modular potentials and symmetry breaking } & 11\end{array}$

$\begin{array}{lll}4.3 & \text { Modular Eisenstein series } & 12\end{array}$

5 Observables in modular inflation $\quad 12$

$\begin{array}{lll}5.1 & \text { Modular inflation parameters } & 13\end{array}$

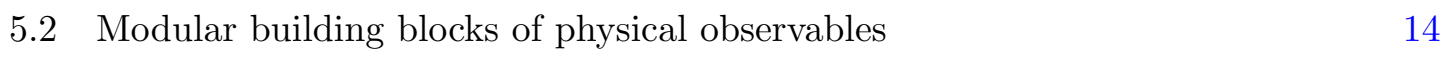

$\begin{array}{ll}\text { 5.3 The almost holomorphic modularity of CMB observables } & 16\end{array}$

$\begin{array}{llr}6 & \text { Dynamics via transfer functions } & 17\end{array}$

$\begin{array}{lll}7 & j \text {-inflation } & 18\end{array}$

8 Observables of $\boldsymbol{j}$-inflation $\quad 20$

8.1 Modular form of observables 21

$8.2 j$-inflation observables and the PLANCK probe 22

9 Conclusion $\quad 24$

\section{Introduction}

Inflation is a framework defined on the space $\mathcal{F}(M, X)$ of scalar field theories defined by field multiplets $\phi^{I}, I=1, \ldots, n$ on the spacetime manifold $M$ taking values in the target space $X$. The field space $\mathcal{F}(M, X)$ is in general assumed to be an unstructured set on which dynamical variables and observables are defined, providing the underlying theory spaces of inflation. The dynamics of the background is determined by the metric $G_{I J}\left(\phi^{K}\right)$ on the 
target space and the potential $V\left(\phi^{I}\right)$. While most of the literature assumes that the target space is flat, following $[1,2], X$ can in general have a nontrivial geometry, encoded in the Riemannian metric $G_{I J}$ that defines the kinetic term $\frac{1}{2} G_{I J} g^{\mu \nu} \partial_{\mu} \phi^{I} \partial_{\nu} \phi^{J}$. Early references that admit a nontrivial geometry include [3-6]. Depending on the origin of the model, the action can either be taken to be given by $E$ and $V$ in polynomial form, or more generally can be a non-rational function, such as in the case of brane-induced inflation.

There are several reasons why it is of interest to consider a symmetry based approach to inflation. One motivation arises from the shift-symmetry, an ad-hoc operation that is often invoked because of concerns about higher order corrections to observable parameters of inflationary models. This is reminiscent of duality considerations, which have led in gauge and string theory to the embedding of analogous dualities into larger discrete and continuous groups. A group-theoretic approach is furthermore useful because it endows the inflaton target space with structure that allows the exploration of the inflationary theory space in a more systematic way by using the resulting geometry as an organizing principle. Symmetries appear as well in inflation theories based on moduli, sometimes also called modular inflation, in which inflation is driven by some of the moduli that appear in many fundamental theories [7]. Such moduli based models can provide special cases of the framework developed here.

The idea of automorphic inflation is to consider the space of the inflationary multifield space defined by theories that are invariant under infinite discrete symmetry groups that contain the inflaton shift symmetry. This leads to the notion of automorphic inflation as a structured framework of multifield inflation [8,9]. An immediate consequence of the existence of such symmetries is that the space of automorphic field theories acquires a foliation, with leaves that are specified by numerical characteristics, defined in terms of the group theoretic and automorphic structure, specifically the underlying continuous group $G$, the discrete group $\Gamma$ in $G$ that extends the shift symmetry, and the types of the automorphic forms that define the building blocks of the inflaton potential.

The simplest case of automorphic inflation is obtained when the discrete group is the modular group, leading to the special case of modular inflation, in which case the continuous group $G$ is fixed to be the Möbius group $\mathrm{SL}(2, \mathbb{R})$ and the discrete group is a subgroup $\Gamma \subset \mathrm{SL}(2, \mathbb{Z}) .{ }^{1}$ The theory of modular forms has been developed over the past century in greatest detail for groups $\Gamma$ that are of congruence type with some level $N$, but more general groups of Fuchsian type are also possible. The potential $V\left(\phi^{I}\right)$ is constructed in terms of modular forms $f_{i}$ of weight $w_{i}$ relative to $\Gamma(N)$. The resulting theory space of modular inflation is thus determined by the numerical characteristics $\left(N, w_{i}\right)$. The kinetic term of all modular inflation models is determined by the Poincaré metric of the upper halfplane, leading to a conformally flat target space for the inflaton. The invariance of this metric under the continuous group of Möbius transformations leads via the introduction of the inflaton potential to a breaking of the group $\operatorname{PSL}(2, \mathbb{R})$ to the discrete group of modular transformations $\operatorname{PSL}(2, \mathbb{Z})$, or subgroups thereof. The energy scale of this breaking is determined by CMB constraints, leading to a weakly broken symmetry in the case considered here.

\footnotetext{
${ }^{1}$ In the case of inflation driven by two or more moduli fields the presence of appropriate symmetries places such models in the framework of modular, and more generally, automorphic inflation.
} 
Automorphic inflation was briefly outlined in [8], and the general framework was described in more detail in [9], with emphasis on the automorphic side of the theory. The purpose of the present paper is to focus in more detail on the class of modular inflation and the particular model of $j$-inflation. The phenomenological analysis of general modular inflation involves derivatives of the potential, hence derivatives of modular forms. However, derivatives of modular forms are not modular forms, raising the issue of the modular structure of observables in this framework. It is shown that the basic CMB observables are in general determined not by modular forms per se but by almost holomorphic modular forms. The phenomenological analysis of the specific model of $j$-inflation, introduced in [8], is compared to observational constraints from the microwave anisotropy. Sections 2 and 3 briefly introduce the general multifield inflationary background dynamics and perturbation theory, with emphasis on a closed system of equations for the isocurvature perturbation described by an antisymmetric rank two tensor $S^{I J}$. This generalizes an earlier result obtained in the special case of flat two-field inflation to an arbitrary number of fields with a general curved target space. Sections 4 and 5 consider modular inflation, with focus on the general structure of physical observables in this framework, showing in particular that they are modular invariant in a generalized sense. Section 6 briefly describes the integration of the inflationary evolution in terms of the transfer function, and sections 7 and 8 analyze the specific model of $j$-inflation. The final section presents some conclusions.

\section{Multifield dynamics}

Automorphic inflation as a group theoretic framework for multifield inflation involves field spaces that are obtained as coset spaces of continuous groups, hence are curved. The specialization to modular inflation leads to an extensive class of two-field models with conformally flat target spaces. In this section some essential features of these theories are described. There are different ways to encode the dynamics of the adiabatic and isocurvature perturbations. In the following the comoving curvature perturbation $\mathcal{R}$ will be adopted as the adiabatic mode, while the isocurvature perturbations are encoded in an antisymmetric tensor denoted by $S^{I J}$. The focus on the latter is suggested by the dynamics of $\mathcal{R}$, leading to an isocurvature dynamics different from the usual dynamics based on projections of the Sasaki-Mukhanov variables. While the geometry in automorphic inflation is derived from the structure of the underlying Lie group $G$ and certain subgroups, it is best to leave the metric of the inflaton field space arbitrary and the number of fields of the inflaton multiplet $\phi^{I}$ unconstrained, so as to indicate the general features of the framework. Modular inflation is then recovered by setting $G=\mathrm{SL}(2, \mathbb{R})$ and considering the field space $G / K$ with the compact subgroup $K=\mathrm{SO}(2, \mathbb{R})$, which is isomorphic to the complex upper halfplane.

\subsection{Scalar field dynamics}

The scalar field action is considered to be of the type

$$
\mathcal{A}\left[\phi^{I}, G_{I J}, g_{\mu \nu}\right]=\int d^{4} x \sqrt{-g}\left(\frac{M_{\mathrm{Pl}}^{2}}{2} R-\frac{1}{2} G_{I J} g^{\mu \nu} \partial_{\mu} \phi^{I} \partial_{\nu} \phi^{J}-V\left(\phi^{I}\right)\right),
$$


where the spacetime metric has the signature $(-,+,+,+)$ and $M_{\mathrm{Pl}}=1 / \sqrt{8 \pi G_{N}}$ is the reduced Planck mass associated to Newton's constant $G_{N}$. The target space spanned by the inflaton fields $\phi^{I}, I=1, \ldots, N$, in general has a non-trivial geometry determined by the metric $G_{I J}$ that is assumed to be Riemannian.

The dynamics of the system $\left(g_{\mu \nu}, G_{I J}, \phi^{I}\right)$ involves the geometry of the target space as well as that of spacetime via the Einstein equations and the Klein-Gordon equation. Assuming that the covariant derivative on the inflaton space is of Levi-Civita type, the Euler-Lagrange form of the latter takes the form

$$
\square_{g} \phi^{I}+\Gamma_{J K}^{I} g^{\mu \nu} \partial_{\mu} \phi^{J} \partial_{\nu} \phi^{K}-G^{I J} V_{, J}=0,
$$

where $\square_{g}=\frac{1}{\sqrt{-g}} \partial_{\mu} \sqrt{-g} g^{\mu \nu} \partial_{\nu}$ is the d'Alembert operator and $\Gamma_{J K}^{I}$ are the target space Christoffel symbols. This Klein-Gordon equation can be written in terms of a covariant derivative $D_{\mu}$, which can be viewed as a combination of the spacetime Koszul connection

$$
\nabla_{\partial_{\mu}} \partial^{\kappa} \phi^{I}=\partial_{\mu} \partial^{\kappa} \phi^{I}+\Gamma_{\mu \nu}^{\kappa} \partial^{\nu} \phi^{I}
$$

and a contribution of the curved target space

$$
D_{\mu} \partial^{\kappa} \phi^{I}=\nabla_{\partial_{\mu}} \partial^{k} \phi^{I}+\left(\partial_{\mu} \phi^{J}\right) \Gamma_{J K}^{I} \partial^{\kappa} \phi^{K}
$$

as

$$
D_{\mu}\left(\partial^{\mu} \phi^{I}\right)-G^{I J} V_{, J}=0
$$

\subsection{Curved target background dynamics}

The dynamics of the background is assumed to belong to the class of theories characterized by the action (2.1). The Klein-Gordon equation is given by

$$
D_{t} \dot{\phi}^{I}+3 H \dot{\phi}^{I}+G^{I J} V_{, J}=0
$$

where the dot indicates the derivative with respect to $t$ and $D_{t}$ is the covariant derivative on the target space, defined for vector fields $W^{I}$ in terms of the connection coefficients $\Gamma_{I J}^{K}$ as

$$
D_{t} W^{I}=\partial_{t} W^{I}+\Gamma_{J K}^{I} \dot{\phi}^{J} W^{K}
$$

Here the $\Gamma_{I J}^{K}$ are universally assumed to be the Christoffel symbols determined by the Levi-Civita connection. With $\partial_{t}=\left(\partial_{t} \phi^{I}\right) \partial_{I}$ this translates into $D_{t} W^{I}=\dot{\phi}^{J} \nabla_{\partial_{J}} W^{I}$, where $\nabla_{\partial_{I}}$ is the Koszul derivative of the target space

$$
\nabla_{\partial_{I}} W^{J}=\partial_{I} W^{J}+\Gamma_{I K}^{J} W^{K}
$$

The background equations constraining the Hubble parameter $H=\dot{a} / a$ are given in the Newtonian gauge with metric

$$
d s^{2}=-d t^{2}+a^{2}(t) \gamma_{i j} d x^{i} d x^{j}
$$


where in the present paper the spatial metric $\gamma_{i j}$ is chosen to be flat for simplicity, by the two Friedman-Lemaitre equations

$$
H^{2}=\frac{\rho}{3 M_{\mathrm{Pl}}^{2}}
$$

and

$$
2 \frac{\ddot{a}}{a}+H^{2}=-\frac{p}{M_{\mathrm{Pl}}^{2}},
$$

where the density $\rho$ and the pressure $p$ of the isotropic fluid tensor

$$
T_{\mu \nu}=(\rho+p) u_{\mu} u_{\nu}+p g_{\mu \nu}
$$

are given by

$$
\begin{aligned}
\rho & =\frac{1}{2} G_{I J} \dot{\phi}^{I} \dot{\phi}^{J}+V\left(\phi^{I}\right) \\
p & =\frac{1}{2} G_{I J} \dot{\phi}^{I} \dot{\phi}^{J}-V\left(\phi^{I}\right) .
\end{aligned}
$$

It is useful to note that the variation of the Hubble parameter is given by

$$
\dot{H}=-\frac{1}{2 M_{\mathrm{Pl}}^{2}}\left(G_{I J} \dot{\phi}^{I} \dot{\phi}^{J}\right) \text {. }
$$

In all these background equations the functions on field space are functions of the background fields $\phi^{I}(t)$.

\subsection{Slow-roll dynamics}

In the slow-roll approximation the kinetic energy is assumed to be small compared to the potential energy, $\rho_{\text {kin }} \ll V$, and the acceleration of the inflaton is assumed to be small as well. More precisely, it is conventional to introduce the parameters

$$
\begin{aligned}
& \epsilon:=-\frac{\dot{H}}{H^{2}}=\frac{3 \dot{\sigma}^{2}}{\dot{\sigma}^{2}+2 V} \\
& \eta:=\frac{1}{H \epsilon} \frac{d \epsilon}{d t}
\end{aligned}
$$

where the background dynamics has been used and $\dot{\sigma} \equiv v=\sqrt{G_{I J} \dot{\phi}^{I} \dot{\phi}^{J}}$ denotes the background inflaton speed, in the notation of $[10,11]$, respectively. The parameter $\eta$ can be written as [12]

$$
\eta=2 \epsilon+\frac{2}{H} \frac{\dot{\phi}^{I} D_{t} \dot{\phi}_{I}}{G_{K L} \dot{\phi}^{K} \dot{\phi}^{L}}=2 \epsilon+\frac{2}{H} \frac{\sigma^{I} D_{t} \dot{\phi}_{I}}{\dot{\sigma}}
$$

which shows that the slow-roll approximation constraint $\eta \ll 1$ translates into a small projection of the acceleration vector onto the inflaton velocity.

The slow-roll form of the background equation can then be used to eliminate the background field velocity

$$
\dot{\phi}^{I}=-\frac{G^{I J} V_{, J}}{3 H}
$$

and the slow-roll form of the first Friedman-Lemaitre equation reduces to

$$
H^{2}=\frac{V}{3 M_{\mathrm{Pl}}^{2}} .
$$




\section{Perturbed multifield inflation}

While $j$-inflation is an example of two-field inflation, it is conceptually more transparent to leave the number of fields in the following brief discussion of inflationary perturbation theory unrestricted. For scalar field theory with flat target spaces this has been considered in many references, including $[1,2,10,13]$, and reviews can be found in $[14,15]$. A comprehensive review for curved target space inflation has not yet been written, but the references [11, 16-26] contain brief descriptions of some aspects of multifield inflation with a non-trivial field space geometry, and $[12,19-22,27,28]$ are concerned with covariant formulations that extend the construction of [29].

The metric perturbations are conventionally parametrized as

$$
d s^{2}=-(1+2 \varphi) d t^{2}+2 a B_{, i} d x^{i} d t+a^{2}\left((1-2 \psi) \delta_{i j}+2 E_{, i j}\right) d x^{i} d x^{j},
$$

where $\varphi=\psi$ in the absence of anisotropic stresses, and different gauges are defined via the vanishing of some of these perturbations. The notation adopted in (3.1) is close to that of the reviews [30, 31].

The inflationary dynamics has been constrained over the past two decades by the CMB satellite probes COBE, WMAP and PLANCK, providing experimental results for some observational variables associated to the gravitational and inflaton perturbations at the percent level, and non-trivially bounding others.

\subsection{Dynamics of perturbations}

A commonly adopted perturbation is the comoving curvature perturbation, defined for general fluids in the Newton gauge as $[16,32,33]$

$$
\mathcal{R}=H \delta u-\psi,
$$

where $\psi$ is the spatial metric perturbation and $\delta u$ is obtained from the divergence part of the energy momentum tensor perturbation $\delta T_{0 i}=-(\rho+p) \delta u_{i}$ with $\delta u_{i}=\partial_{i} \delta u+u_{i}$ as

$$
\delta T_{0 i}^{\operatorname{div}}=-(\rho+p) \partial_{i} \delta u
$$

The above definition is often written in a different form by introducing $\delta q=(\rho+p) \delta u$ and writing $\mathcal{R}$ in terms of $\delta q$. In multifield scalar field theory with curved target space geometry the perturbation can be expressed in terms of the Sasaki-Mukhanov variables [34, 35]

$$
Q^{I}=\mathcal{Q}^{I}+\frac{\dot{\phi}^{I}}{H} \psi
$$

as

$$
\mathcal{R}=-\frac{H}{\dot{\sigma}} \sigma_{I} Q^{I}
$$

where $\sigma^{I}$ is the normalized inflaton velocity $\sigma^{I}=\dot{\phi}^{I} / \dot{\sigma}$ and $\dot{\sigma}$ is the inflaton speed defined above. Adopting the notation of [22], the variables $\mathcal{Q}^{I}$ denote the covariant form of the field 
perturbations $\delta \phi^{I}(t, \vec{x}):=\phi^{I}(t, \vec{x})-\phi^{I}(t)$ defined in terms of the geodesic path between the perturbed field $\phi^{I}(t, \vec{x})$ and the background field $\phi^{I}(t)$ given by [29]

$$
\delta \phi^{I}=\mathcal{Q}^{I}-\frac{1}{2} \Gamma_{J K}^{I} \mathcal{Q}^{J} \mathcal{Q}^{K}+\cdots
$$

For the time evolution of $\mathcal{R}$ we find here in terms of rank two tensors the equation

$$
\dot{\mathcal{R}}=\frac{H}{\dot{H}} \frac{1}{a^{2}} \Delta \psi+\frac{1}{\dot{\sigma}} S^{I J} W_{I J},
$$

where

$$
S^{I J}:=\frac{H}{\dot{\sigma}}\left(\sigma^{I} Q^{J}-\sigma^{J} Q^{I}\right),
$$

and the gradient tensor is defined as

$$
W_{I J}:=\sigma_{I} V_{, J}-\sigma_{J} V_{, I}
$$

This suggests identifying the dimensionless variables $S^{I J}$ as the fundamental perturbations that source the large scale time evolution of $\mathcal{R}$. They will be referred to as the rank 2 tensor of the isocurvature perturbations.

In terms of the isocurvature variables $S^{I J}$ adopted here as the basic nonadiabatic perturbations, the slow-roll approximation of the $\mathcal{R}$-dynamics on large scales is given by

$$
\dot{\mathcal{R}}=-2 H \eta_{I K} \sigma^{K} \sigma_{J} S^{I J},
$$

where the slow-roll parameter $\eta_{I J}$ is defined as

$$
\eta_{I J}:=M_{\mathrm{Pl}}^{2} \frac{V_{; I J}}{V} .
$$

The time evolution of the isocurvature perturbation tensor in this approximation is given by

$$
D_{t} S^{I J}=2 H\left(\eta_{K L} \sigma^{K} \sigma^{L}-\epsilon\right) S^{I J}+H\left(\eta_{K L} G^{K[I} S^{J] L}-\frac{\epsilon}{3} M_{\mathrm{Pl}}^{2} \sigma^{[I} R_{K L M}^{J]} \sigma^{K} S^{L M}\right),
$$

where the brackets $U^{[I} V^{J]}$ indicate antisymmetrization without the conventional factor of $1 / 2$, and $D_{t}$ is the covariant derivative acting on the contravariant tensor $S^{I J}$. The differential equations (3.10) and (3.12) form a closed system of evolution equations for the comoving curvature perturbation $\mathcal{R}$ and the isocurvature perturbations $S^{I J}$ which shows how in the general multifield case the latter mix during the evolution and how they couple to the curvature tensor. Equation (3.10) shows that the adiabatic perturbation remains constant on large scales if the vector defined by the slow-roll contraction $\eta_{I J} \sigma^{J}$ of the normalized inflaton velocity $\sigma^{I}$ is orthogonal to the isocurvature contraction $S^{I J} \sigma_{J}$ of $\sigma^{I}$. Alternatively one can view the r.h.s. as a quadratic form

$$
D_{I J}:=G_{I K} S^{K L} \eta_{L J}
$$

defined on the tangent space of the target manifold. The system (3.10), (3.12) generalizes the flat target two-field dynamics of ref. [13] to curved field spaces of arbitrary dimension. 
The power spectrum associated to a dimensionless perturbation, in the following generically denoted by $\mathcal{O}(t, \vec{x})$, is defined in terms of the correlator as

$$
\left\langle\mathcal{O}(t, \vec{k}) \mathcal{O}^{\prime}\left(t, \vec{k}^{\prime}\right)\right\rangle=:(2 \pi)^{3} \delta^{(3)}\left(\vec{k}-\vec{k}^{\prime}\right) P_{\mathcal{O O}}(k) .
$$

with the associated dimensionless power spectrum defined as

$$
\mathcal{P}_{\mathcal{O O}}:=\frac{k^{3}}{2 \pi^{2}} P_{\mathcal{O O}^{\prime}}
$$

In the present case the variables $\mathcal{O}, \mathcal{O}^{\prime}$ are given by the perturbations $\left(\mathcal{R}, S^{I J}\right)$.

The dynamics of $\mathcal{R}$ identifies the isocurvature tensor contraction as the essential source of the large scale behavior of the adiabatic perturbation. We associate to the tensor $S^{I J}$ a dimensionless isocurvature scalar such that its power spectrum at horizon crossing is identical to that of $\mathcal{R}$ by introducing

$$
\mathcal{S}=-\frac{1}{2 \alpha \dot{\sigma}} S^{I J} W_{I J}
$$

where $\alpha$ is the absolute value of the normalized acceleration vector $\alpha^{I}:=D_{t} \sigma^{I}$. Per construction the power spectrum $\mathcal{P}_{\mathcal{S S}}$ of $\mathcal{S}$ as defined in (3.14) is identical to that of $P_{\mathcal{R} R}$, given in its dimensionless form by

$$
\mathcal{P}_{\mathcal{R} \mathcal{R}}=\left(\frac{H}{2 \pi}\right)^{2}\left(\frac{H}{\dot{\sigma}}\right)^{2},
$$

while the cross-correlation power vanishes at horizon crossing

$$
\mathcal{P}_{\mathcal{S S}}=\mathcal{P}_{\mathcal{R} \mathcal{R}}, \quad \mathcal{P}_{\mathcal{R S}}=0 .
$$

\subsection{The slow-roll form of the power spectrum}

The slow-roll approximation of the adiabatic power spectrum $\mathcal{P}_{\mathcal{R} \mathcal{R}}$ can be expressed either in terms of the potential or in terms of the slow-roll parameters $\eta_{I J}$ introduced above and

$$
\epsilon_{I}=M_{\mathrm{Pl}} \frac{V_{, I}}{V},
$$

which resolves the parameter $\epsilon$ defined above. Using the resulting $\dot{\sigma}^{2}=(V / 3) G^{I J} \epsilon_{I} \epsilon_{J}$ leads to the power spectrum at horizon crossing as

$$
\mathcal{P}_{\mathcal{R} \mathcal{R}}=\frac{1}{12 \pi^{2} M_{\mathrm{Pl}}^{4}} \frac{V}{G^{I J} \epsilon_{I} \epsilon_{J}} .
$$

As noted above, $\mathcal{P}_{\mathcal{R S}}=0$ and $\mathcal{P}_{\mathcal{S S}}=\mathcal{P}_{\mathcal{R} \mathcal{R}}$ at horizon crossing

The spectral indices $n_{\mathcal{O O}}$ are obtained from the power spectrum $\mathcal{P}_{\mathcal{O O}^{\prime}}$ as

$$
n_{\mathcal{O O}^{\prime}}=1+\frac{d \ln \mathcal{P}_{\mathcal{O O}}}{d \ln k} .
$$

The shift by unity is conventional for the adiabatic perturbation, but is not always adopted for isocurvature perturbations in the literature. The slow-roll form of the power spectrum above then leads to the spectral index

$$
n_{\mathcal{R} \mathcal{R}}=1-3 G^{I J} \epsilon_{I} \epsilon_{J}+2 \frac{\eta_{I J} \epsilon^{I} \epsilon^{J}}{G^{K L} \epsilon_{K} \epsilon_{L}} .
$$

The constraints on $n_{\mathcal{O O}^{\prime}}$ obtained by WMAP [36] and PLANCK $[37,38]$ therefore restrict the shape of the potentials. 


\subsection{The tensor-to-scalar ratio}

Gravitational waves play an important role in constraining the viable part of the inflationary theory space. While no primordial signal has been detected, satellite probes like WMAP and PLANCK have led to upper bounds that models have to satisfy. Conventionally, these bounds are formulated in terms of the tensor-to-scalar ratio $r$, which is constructed from the tensor power spectrum [39]

$$
\mathcal{P}_{T}=\frac{2}{\pi^{2}}\left(\frac{H}{M_{\mathrm{Pl}}}\right)^{2}
$$

and the scalar power spectrum. In multifield inflation different forms for $r$ have been considered. A convenient way to define $r$ is as the ratio of the tensor-to-adiabatic scalar amplitude

$$
r:=\frac{\mathcal{P}_{T}}{\mathcal{P}_{\mathcal{R} R}}
$$

which leads to the slow-roll parameter form

$$
r=8 G^{I J} \epsilon_{I} \epsilon_{J}
$$

The tensor spectral index, defined via the ansatz

$$
\mathcal{P}_{T}(k)=\mathcal{P}_{T}\left(k_{p}\right)\left(\frac{k}{k_{p}}\right)^{n_{T}}
$$

can be written in terms of the slow-roll parameters as

$$
n_{T}=-G^{I J} \epsilon_{I} \epsilon_{J}
$$

leading with eq. (3.25) to the $\left(r, n_{T}\right)$-relation

$$
r=-8 n_{T}
$$

in the slow-roll approximation. This relation is affected by the transfer functions, as discussed further below.

\section{Modular inflation}

In this section the framework of automorphic inflation is specialized to the case of modular inflation, a particular class of two-field scalar field theories coupled to gravity with a nontrivial target space geometry, which is of coset type $G / K$, where $G$ is a Lie group and $K \subset G$ is a maximal compact subgroup. The general framework was introduced in [8] and its structure described in more detail in [9] in the higher rank case.

Classical modular forms $[40,41]$ were introduced in the second half of the $19^{\text {th }}$ century as functions on the complex upper halfplane because this space is mapped to itself by the modular group $\mathrm{SL}(2, \mathbb{Z})$. The general concept was introduced by Klein [42] in the context of various discrete subgroups $\Gamma \subset \mathrm{SL}(2, \mathbb{Z})$. Thinking about forms in this way is computationally useful, but conceptually not the most illuminating approach, and it is 
most advantageous to have both the domain theoretic and the group theoretic formulations available. Such a framework is described in refs. $[8,9]$. The result is that in the modular context the group theoretic set-up is given by the pairs of groups $(G, \Gamma)$, where $G=\mathrm{SL}(2, \mathbb{R})$ is semisimple. The domain theoretic structure is obtained by considering a maximal compact subgroup $K \subset G$, which in this case is the rotation group $K=\mathrm{SO}(2, \mathbb{R})$, both of which act via the Möbius transformation. More details can be found in [9].

The discrete groups can be of Fuchsian type but the most well-developed theory is that of different types of congruence groups $\Gamma_{N} \subset \mathrm{SL}(2, \mathbb{Z})$, where the level $N$ determines the defining congruence constraint. For Hecke groups the matrices

$$
\gamma=\left(\begin{array}{ll}
a & b \\
c & d
\end{array}\right) \in \Gamma_{0}(N) \subset \operatorname{SL}(2, \mathbb{Z})
$$

satisfy the constraint $c \equiv 0(\bmod N)$. Other possibilities include groups usually denoted by $\Gamma_{1}(N)$ and $\Gamma(N)$. These will collectively be denoted as $\Gamma_{N}$ in the following.

\subsection{Kinetic term}

The bounded domain $\mathcal{H}$ is two-dimensional, which implies via the Bianchi identity that the Riemann curvature tensor takes the form

$$
R_{I J K L}=\mathcal{K}\left(G_{I K} G_{J L}-G_{I L} G_{J K}\right),
$$

where $\mathcal{K}=R / 2$ is the Gaussian curvature expressed in terms of the Ricci scalar $R$. The metric on $\mathcal{H}$ induced by the Cartan-Killing form $B$ on the Lie algebra $\mathfrak{s l}(2, \mathbb{R})$ is the Poincaré metric

$$
d s^{2}=\frac{d \tau d \bar{\tau}}{(\operatorname{Im} \tau)^{2}}=\frac{d x^{2}+d y^{2}}{y^{2}} .
$$

The field theoretic form of the Poincaré metric

$$
d s^{2}=G_{I J} d \phi^{I} d \phi^{J}=\frac{\mu^{2}}{\left(\phi^{2}\right)^{2}} \delta_{I J} d \phi^{I} d \phi^{J}
$$

leads to the non-vanishing Christoffel symbols

$$
\Gamma_{11}^{2}=-\Gamma_{22}^{2}=-\Gamma_{12}^{1}=\frac{1}{\phi^{2}}=\frac{1}{\mu(\operatorname{Im} \tau)} .
$$

The curvature tensor $R_{I J K L}$ has only one independent component

$$
R_{1212}=-\frac{1}{\mu^{2}(\operatorname{Im} \tau)^{4}},
$$

leading to the curvature scalar

$$
R=2 G^{11} G^{22} R_{1212}=-\frac{2}{\mu^{2}} .
$$

Thus the space $\left(\mathcal{H}, d s^{2}\right)$ with the metric (4.3) has constant negative Gaussian curvature $\mathcal{K}=-1 / \mu^{2}$. 
The kinetic term, written in multifield notation as $G_{I J} g^{\mu \nu} \partial_{\mu} \phi^{I} \partial_{\nu} \phi^{J} / 2$ with $I, J=1,2$ is invariant under the group defined by the linear fractional transformations

$$
g \tau=\frac{a \tau+b}{c \tau+d}
$$

where

$$
g=\left(\begin{array}{ll}
a & b \\
c & d
\end{array}\right), \quad \operatorname{det} g>0
$$

are matrices with real entries. These Möbius transformations map the upper halfplane to itself and define the continuous symmetry group of the free modular theory. Since the center of $\operatorname{SL}(2, \mathbb{R})$ acts trivially the continuous invariance group is given by $\operatorname{PSL}(2, \mathbb{R})$.

The metric (4.4) has a structure that is reminiscent of the metrics encountered in single field pole inflation considered in refs. $[43,44]$ in that it has a double pole for vanishing $\phi^{2}$. In modular inflation the boundary $\operatorname{Im}(\tau)=0$ however is not part of the domain of the inflaton field and neither the metric nor the potential are defined on the real axis of the complex plane.

\subsection{Modular potentials and symmetry breaking}

The action of modular inflation can be written as

$$
\mathcal{A}_{\text {mod }}=\int d^{4} x \sqrt{-g}\left(\frac{M_{\mathrm{Pl}}^{2}}{2} R-\frac{1}{2} G_{\tau \bar{\tau}} g^{\mu \nu} \partial_{\mu} \tau \partial_{\nu} \bar{\tau}-V(F(\tau))\right),
$$

where $G_{\tau \bar{\tau}}$ is the complex form of the Poincaré metric. The potentials of modular two-field theory are defined in terms of modular forms that descend from the group to the upper halfplane. The general construction of the inflaton target space $X$ in terms of the Lie group $G$ has been described in detail in [9].

Modular forms on the upper halfplane $\mathcal{H}$ are induced by the group function $\Phi$ : $\mathrm{SL}(2, \mathbb{R}) \longrightarrow \mathbb{C}$ via the 1-cocycle

$$
J(g, \tau)=(c \tau+d), \quad g=\left(\begin{array}{ll}
a & b \\
c & d
\end{array}\right)
$$

as

$$
f(\tau)=(c \tau+d)^{w} \Phi(g),
$$

where $\tau=g i$. They are defined with respect to discrete subgroups $\Gamma_{N}$ of the modular group $G(\mathbb{Z})=\operatorname{SL}(2, \mathbb{Z})$ and are characterized by the level $N$ of the subgroups $\Gamma_{N}$, their weights $w$, and by a character $\epsilon_{N}$ via their transformation behavior, which for $\gamma \in \Gamma_{N}$ and the action given by the discrete Möbius transformation $\gamma \tau=(a \tau+b) / c \tau+d)$ is defined to be

$$
f(\gamma \tau)=\epsilon_{N}(d)(c \tau+d)^{w} f(\tau)
$$

The potential $V\left(\phi^{I}\right)$ is defined in terms of modular functions $F(\tau)$ on the upper halfplane, as well as some function $\Phi(F)$ as

$$
V=\Lambda^{4} \Phi(F, \bar{F})
$$


where $\Lambda^{4}$ is an energy scale and $F$ and $\Phi$ are dimensionless. A simple class of functions $\Phi$ is given by powers of the norm function, leading to

$$
V_{p}:=\Lambda^{4}|F(\tau)|^{2 p}
$$

In the present paper the focus will be on the $p=1$ case. The modular functions $F$ can in general be viewed as a discrete subgroup of the modular group $\operatorname{SL}(2, \mathbb{Z})$, but the most detailed theory of forms has been formulated for congruence groups $\Gamma_{N}$ of various types with level $N$. The introduction of the potential thus breaks the continuous Möbius group of the previous subsection to the discrete subgroups, which can be written schematically as

$$
\operatorname{PSL}(2, \mathbb{R}) \longrightarrow \Gamma_{N} \subset \operatorname{PSL}(2, \mathbb{Z})
$$

In $j$-inflation, the example considered further below, the Möbius symmetry is weakly broken since the constraints from the CMB determine the energy scale $\Lambda$ to be much lower than the Planck scale.

\subsection{Modular Eisenstein series}

As in the automorphic case, Eisenstein series play a key role for modular forms of arbitrary weight because for the full modular group they span the subspace complementary to the cusp forms. Holomorphic Eisenstein series are obtained by following the general construction briefly outlined in the general case in the previous subsection. The details of how to obtain from the group theoretic Eisenstein series on $G=\mathrm{SL}(2, \mathbb{R})$ the classical Eisenstein functions $E_{w}(\tau)$ on the upper halfplane, given in terms of the divisor function

$$
\sigma_{w}(n):=\sum_{d \mid n} d^{w}
$$

the Bernoulli numbers $B_{w}$, and $q=e^{2 \pi i \tau}$ with $\tau \in \mathcal{H}$, as

$$
E_{w}(\tau)=1-\frac{2 w}{B_{w}} \sum_{n} \sigma_{w-1}(n) q^{n}
$$

can be found in ref. [9].

There are different ways to obtain the values for $B_{w}$, for example via the generating function $x /\left(e^{x}-1\right)=\sum_{m=0}^{\infty} B_{m} x^{m} / m$ !, or in terms of the Riemann zeta function via Euler's formula as $B_{w}=-2 w ! \zeta(w) /(2 \pi i)^{w}$. For $w>2$ these functions are modular. In the case of $j$-inflation the forms of weight 2, 4, 6 are relevant and with Euler's results for $\zeta(2), \zeta(4)$ and $\zeta(6)[45]$ the zeta function relation leads to $B_{2}=1 / 6, B_{4}=-1 / 30, B_{6}=1 / 42$. These ingredients will be used below to define $j$-inflation.

\section{Observables in modular inflation}

It is shown in this section that for general modular invariant inflation the physical observables are determined by modular forms that are almost holomorphic, but in general 
not holomorphic. The explicit form of the spectral index and the tensor-to-scalar ratio is determined in terms of the defining modular function $F$ of the inflationary potential.

For two-field inflation the dynamical system introduced above simplifies considerably because there is only one independent isocurvature perturbation. The general adiabatic equation (3.10) takes the form

$$
\dot{\mathcal{R}}=-2 H \sigma^{K} \eta_{K[1} \sigma_{2]} S^{12},
$$

which in the case of modular inflation simplifies further because the metric is conformally flat $G_{11}=G_{22}=G$, resulting in

$$
\dot{\mathcal{R}}=2 H G \eta_{\sigma s} S^{12}
$$

with

$$
\eta_{\sigma s}=\left(\eta_{22}-\eta_{11}\right) \sigma^{1} \sigma^{2}+\eta_{12}\left(\left(\sigma^{1}\right)^{2}-\left(\sigma^{2}\right)^{2}\right) .
$$

The isocurvature equation (3.12) reduces to

$$
D_{t} S^{12}=H\left(\eta_{\sigma \sigma}-\eta_{s s}+2 \epsilon\left(\frac{1}{3} M_{\mathrm{Pl}}^{2} \mathcal{K}-1\right)\right) S^{12},
$$

where the abbreviations $\eta_{\sigma \sigma}=\eta_{I J} \sigma^{I} \sigma^{J}$ and

$$
\eta_{s s}=\eta_{11}\left(\sigma^{2}\right)^{2}-2 \eta_{12} \sigma^{1} \sigma^{2}+\eta_{22}\left(\sigma^{1}\right)^{2}
$$

are covariant objects obtained from (3.11). This specialization of the general dynamics of the system $\left(\mathcal{R}, S^{I J}\right)$ derived above extends the discussion of ref. [13] for two-field inflation with a flat field space metric to curved target spaces.

\subsection{Modular inflation parameters}

The inflationary analysis considered in section 3 involves the geometry of the potential. General expressions for the observables in modular inflation thus involve derivatives of modular forms. For a potential of modular functions

$$
V=\Lambda^{4}|F|^{2}
$$

for a modular function $F$ the slow-roll parameters $\epsilon_{I}$ defined in eq. (3.19) take the form

$$
\epsilon_{I}=i^{I-1} \frac{M_{\mathrm{Pl}}}{\mu}\left(\frac{F^{\prime}}{F}+(-1)^{I-1} \frac{\bar{F}^{\prime}}{\bar{F}}\right)
$$

and the acceleration of the scale parameter is directly determined by the behavior of

$$
\epsilon_{V}:=\frac{1}{2} G^{I J} \epsilon_{I} \epsilon_{J}=2 \frac{M_{\mathrm{Pl}}^{2}}{\mu^{2}}(\operatorname{Im} \tau)^{2}\left|\frac{F^{\prime}}{F}\right|^{2} .
$$

These parameters determine the tensor-to-adiabatic scalar ratio and part of the spectral index. 
The remaining ingredient of $n_{\mathcal{R} \mathcal{R}}$ is the parameter matrix $\eta_{I J}$ defined in (3.11). Decomposing the covariant derivative into its flat and Christoffel contributions

$$
\eta_{I J}=\eta_{I J}^{\mathrm{f}}+\eta_{I J}^{\Gamma}
$$

leads to the flat part

$$
\eta_{I J}^{\mathrm{fl}}=-i^{I+J} \frac{M_{\mathrm{Pl}}^{2}}{\mu^{2}}\left(\frac{F^{\prime \prime}}{F}-\left((-1)^{I}+(-1)^{J}\right)\left|\frac{F^{\prime}}{F}\right|^{2}+(-1)^{I+J} \frac{\bar{F}^{\prime \prime}}{\bar{F}}\right),
$$

while the Christoffel term $\eta_{I J}^{\Gamma}=-M_{\mathrm{Pl}} \Gamma_{I J}^{K} \epsilon_{K}$ is given by

$$
\eta_{I J}^{\Gamma}=-\frac{M_{\mathrm{Pl}}^{2}}{\mu^{2}} \frac{i^{I+J-1}}{\operatorname{Im} \tau}\left(\frac{F^{\prime}}{F}+(-1)^{I+J-1} \frac{\bar{F}^{\prime}}{\bar{F}}\right) .
$$

For modular inflation models in which the curvature contribution is small, like in $j$-inflation, the flat limit provides a very good approximation to the full result.

\subsection{Modular building blocks of physical observables}

Modular functions $F$ can be written as quotients of modular forms, hence the computation of $F^{\prime}$ reduces to the computation of $f^{\prime}$ for modular forms of some arbitrary weight $w$. However, the derivative of a modular form is not a modular form. This raises the issue of what precisely the modular structure is of the physical observables in modular invariant inflation. The general structure of the derivative takes the form

$$
f^{\prime} \equiv \frac{d f}{d \tau}=2 \pi i\left(\tilde{f}+\frac{w}{12} f E_{2}\right)
$$

where $\tilde{f}$ is a modular form of weight $(w+2)$. The problem to specify the first term in $f^{\prime}$ is nontrivial and was described in some detail in [9]. Very briefly, in the case of the full modular group it is determined by functions $H_{\tau}(z)$ considered in [46]

$$
H_{\tau}(z):=\sum_{n=0}^{\infty} j_{n}(\tau) q^{n}
$$

where $q=e^{2 \pi i z}$ and the functions $j_{n}$ are constructed iteratively via the normalized weight zero Hecke operators $T_{0}(m)$ as $j_{n}(z)=\left.j_{1}(z)\right|_{T_{0}(n)}$, where $j_{1}(z)=j(z)-744$. With

$$
e_{\tau}:=\left\{\begin{array}{cl}
1 / 2 & \text { if } \tau=i \\
1 / 3 & \text { if } \tau=\xi_{3} \\
1 & \text { otherwise }
\end{array}\right\}
$$

the modular form $\tilde{f}$ can be written as

$$
\tilde{f}(z)=-f \sum_{\tau \in \mathcal{F}} e_{\tau} \operatorname{ord}_{\tau}(f) H_{\tau}(z),
$$


where $\operatorname{ord}_{\tau}(f)$ is the vanishing order of $f$, which is constrained by the valence formula

$$
\frac{1}{2} \operatorname{ord}_{\tau=i}(f)+\frac{1}{3} \operatorname{ord}_{\tau=\xi_{3}}(f)+\operatorname{ord}_{\infty}(f)+\sum_{\tau \in \mathcal{F}-i, \xi_{3}} \operatorname{ord}_{\tau}(f)=\frac{w}{12},
$$

where $\xi_{3}=e^{2 \pi i / 3}[47,48]$.

The nonmodularity of $f^{\prime}$ arises from the second term because the Eisenstein series $E_{2}$, defined as in (4.18), transforms under the modular group $\operatorname{SL}(2, \mathbb{Z})$ as

$$
E_{2}(\gamma \tau)=(c \tau+d)^{2} E_{2}(\tau)-\frac{6 i}{\pi} c(c \tau+d)
$$

and therefore is not a modular form. This shows that in the inflationary context the relevant space is not just generated by $E_{4}$ and $E_{6}$, but must also include the Eisenstein series of weight two. $E_{2}$ is the paradigmatic example of a quasimodular form, a notion for which various definitions have been introduced. From a physical point of view it is best to focus on the transformation behavior of these functions under the modular group. The example of $E_{2}$ indicates a structure that is reminiscent of the case of Christoffel symbols, objects with an inhomogeneous transformation behavior, where the tensor behavior is modified by an additional term. Similarly, a quasimodular form transforms like an ordinary modular form, but with additional terms. For a quasimodular form $f^{q}$ of weight $w$ this can be written as

$$
f^{q}(\gamma \tau)=(c z+d)^{w} f(\tau)+(c z+d)^{w} \sum_{m \geq 1}\left(\frac{c}{(c \tau+d)}\right)^{m} f_{m}
$$

where the sum is finite, and $f_{m}$ are holomorphic functions. This definition is more direct, but not less general, than Nahm's definition as given in [49]. The original definition of quasimodular forms was based on the "constant" term of a nearly holomorphic, or almost holomorphic, form as defined by Shimura [50, 51].

The nonmodularity of $f^{\prime}$ a priori induces nonmodular terms in the observables of modular inflation, hence this raises the question what exactly the modular nature of these objects is in modular invariant inflation. For modular functions $F=f / g$ with modular forms $f, g$ of equal weight the derivative turns out to be simple because of a cancellation and we obtain

$$
\frac{F^{\prime}}{F}=2 \pi i\left(\frac{\tilde{f}}{f}-\frac{\tilde{g}}{g}\right)
$$

where $\tilde{f}, \tilde{g}$ are as in (5.12). Thus the first derivative of modular functions are modular forms of weight two. The second derivative $F^{\prime \prime}$ is no longer modular and by using the above formula for the derivative iteratively we find

$$
F^{\prime \prime}=-4 \pi i F^{\prime} \frac{\tilde{g}}{g}-4 \pi^{2} F\left(\frac{\tilde{\tilde{f}}}{f}-\frac{\tilde{\tilde{g}}}{g}\right)+\frac{\pi i}{3} F^{\prime} E_{2}
$$

where $\tilde{\tilde{f}}$ and $\tilde{\tilde{g}}$ are obtained from the derivatives of the modular forms $\tilde{f}$ and $\tilde{g}$. Important for the $\eta_{I J}$-induced terms in the spectral observables is the combination $F^{\prime \prime} / F^{\prime}$. The nonmodular part of this quotient is therefore given by

$$
\left(\frac{F^{\prime \prime}}{F^{\prime}}\right)_{\mathrm{nmod}}=\frac{\pi i}{3} E_{2}
$$


The main result of this and the following discussion is that there are further terms in the modular inflation observables that are induced by the nontrivial geometry of the target space. These terms combine with the nonmodular Eisenstein series $E_{2}$ into a new function that is modular, but not holomorphic, defined as

$$
\widehat{E}_{2}(\tau)=E_{2}(\tau)-\frac{3}{\pi(\operatorname{Im} \tau)} .
$$

The modularity of this function follows from the transformation behavior

$$
\frac{1}{\operatorname{Im} \gamma \tau}=\frac{(c \tau+d)^{2}}{\operatorname{Im} \tau}-2 i c(c \tau+d)=\frac{|c \tau+d|^{2}}{\operatorname{Im} \tau}
$$

The Eisenstein series $\widehat{E}_{2}$ is an example of a nearly holomorphic, or almost holomorphic, modular form. Such a form of weight $w$ for the modular group $\operatorname{SL}(2, \mathbb{Z})$ is a function $f$ on the upper halfplane that is a polynomial in $1 /(\operatorname{Im} \tau)$ with coefficients that are holomorphic functions.

\subsection{The almost holomorphic modularity of CMB observables}

The results above can now be used to address the question raised earlier about the modular structure of the CMB observables. The tensor-to-scalar ration $r$ is determined by the modular form $F^{\prime} / F$ as

$$
r=32 \frac{M_{\mathrm{Pl}}^{2}}{\mu^{2}}(\operatorname{Im} \tau)^{2}\left|\frac{F^{\prime}}{F}\right|^{2}
$$

hence is modular invariant in terms of holomorphic modular forms (and their complex conjugates). The spectral indices involve the second derivatives $F^{\prime \prime}$, hence are not modular in the same sense. From the expressions obtained above for the parameters $\epsilon_{I}$ and $\eta_{I J}$ we obtain for modular inflation the spectral index $n_{\mathcal{R} \mathcal{R}}$ as

$$
n_{\mathcal{R} \mathcal{R}}=1-4 \frac{M_{\mathrm{Pl}}^{2}}{\mu^{2}}(\operatorname{Im} \tau)^{2}\left[2\left|\frac{F^{\prime}}{F}\right|^{2}-\operatorname{Re}\left(\frac{F^{\prime \prime}}{F^{\prime}} \cdot \frac{\bar{F}^{\prime}}{\bar{F}}\right)\right]-4 \frac{M_{\mathrm{Pl}}^{2}}{\mu^{2}}(\operatorname{Im} \tau) \operatorname{Im}\left(\frac{F^{\prime}}{F}\right)
$$

where the final term is induced by the target space metric. The nonmodular contribution to the second term in the square brackets combines with the last term in this equation so that the modularity of the spectral index can be made manifest by writing

$$
n_{\mathcal{R} \mathcal{R}}=1-4 \frac{M_{\mathrm{Pl}}^{2}}{\mu^{2}}(\operatorname{Im} \tau)^{2}\left[2\left|\frac{F^{\prime}}{F}\right|^{2}-\operatorname{Re}\left(\frac{F^{\prime \prime}}{F^{\prime}} \cdot \frac{\bar{F}^{\prime}}{\bar{F}}\right)_{\bmod }+\frac{\pi}{3} \operatorname{Im}\left(\widehat{E}_{2} \frac{\bar{F}^{\prime}}{\bar{F}}\right)\right] .
$$

This completes the derivation of the most important observables for the general framework of modular inflation based on arbitrary modular invariant functions $F$. The weight structure of the inflationary variables can be resolved into their holomorphic and antiholomorphic factors $(w, \bar{w})$. It follows from the above that the slow-roll parameters $\epsilon_{I}$ are determined by forms of weight $(2,0)$ and $(0,2)$, while the parameter $\epsilon_{V}$, which is proportional to $r$, is of weight $(0,0)$. 
The two-field dynamics at the beginning of this section can now be made explicit in terms of the defining modular invariant function $F$ as

$$
\begin{aligned}
& \eta_{\sigma \sigma}=2 \frac{M_{\mathrm{Pl}}^{2}}{\mu^{2}}(\operatorname{Im} \tau)^{2}\left[\left|\frac{F^{\prime}}{F}\right|^{2}+\operatorname{Re}\left(\frac{F^{\prime \prime}}{F^{\prime}} \frac{\bar{F}^{\prime}}{\bar{F}}\right)_{\bmod }-\frac{\pi}{3} \operatorname{Im}\left(\widehat{E}_{2} \frac{\bar{F}^{\prime}}{\bar{F}}\right)\right] \\
& \eta_{s s}=2 \frac{M_{\mathrm{Pl}}^{2}}{\mu^{2}}(\operatorname{Im} \tau)^{2}\left[\left|\frac{F^{\prime}}{F}\right|^{2}-\operatorname{Re}\left(\frac{F^{\prime \prime}}{F^{\prime}} \frac{\bar{F}^{\prime}}{\bar{F}}\right)_{\bmod }+\frac{\pi}{3} \operatorname{Im}\left(\widehat{E}_{2} \frac{\bar{F}^{\prime}}{\bar{F}}\right)\right] \\
& \eta_{\sigma s}=-2 \frac{M_{\mathrm{Pl}}^{2}}{\mu^{2}}(\operatorname{Im} \tau)^{2}\left[\operatorname{Im}\left(\frac{F^{\prime \prime}}{F^{\prime}} \frac{\bar{F}^{\prime}}{\bar{F}}\right)_{\bmod }+\frac{\pi}{3} \operatorname{Re}\left(\widehat{E}_{2} \frac{\bar{F}^{\prime}}{\bar{F}}\right)\right] .
\end{aligned}
$$

These parameters complete the specification of the two-field dynamics for general modular inflation and determine the transfer functions considered in the next section.

\section{Dynamics via transfer functions}

It is useful to note that a system of equations of the form

$$
\begin{aligned}
\dot{\mathcal{R}} & =A H \mathcal{S} \\
\dot{\mathcal{S}} & =B H \mathcal{S}
\end{aligned}
$$

can be integrated formally in terms of transfer functions as

$$
\begin{aligned}
\mathcal{R}(t) & =\mathcal{R}\left(t_{*}\right)+T_{\mathcal{R} \mathcal{S}} \mathcal{S}\left(t_{*}\right) \\
\mathcal{S}(t) & =T_{\mathcal{S} \mathcal{S}} \mathcal{S}\left(t_{*}\right)
\end{aligned}
$$

where

$$
\begin{aligned}
& T_{\mathcal{S S}}\left(t, t_{*}\right)=\exp \left(\int_{t_{*}}^{t} d t^{\prime} B\left(t^{\prime}\right) H\left(t^{\prime}\right)\right) \\
& T_{\mathcal{R S}}\left(t, t_{*}\right)=\int_{t_{*}}^{t} d t^{\prime} A\left(t^{\prime}\right) H\left(t^{\prime}\right) T_{\mathcal{S S}}\left(t^{\prime}, t_{*}\right) .
\end{aligned}
$$

The details of the coefficient functions $A, B$ depend on whether the target space is flat or curved, and on the details of the model. For flat targets they can be expressed in terms of the contractions of the slow-roll parameters $\epsilon_{I}$ and $\eta_{I J}$, as illustrated in the case of two-field inflation in [13]. For curved targets the dynamics involves the curvature of the field manifold, as indicated by eq. (3.12). For two-field inflation this specializes to eq. (5.4), with parameters given in eq. (5.27).

The resulting power spectra of the adiabatic and isocurvature perturbations

$$
\begin{aligned}
& \mathcal{P}_{\mathcal{R \mathcal { R }}}(t)=\left(1+T_{\mathcal{R S}}^{2}\right) \mathcal{P}_{\mathcal{R \mathcal { R }}}\left(t_{*}\right) \\
& \mathcal{P}_{\mathcal{R S}}(t)=T_{\mathcal{R} \mathcal{S}} T_{\mathcal{S} \mathcal{S}} P_{\mathcal{R} \mathcal{R}}\left(t_{*}\right) \\
& \mathcal{P}_{\mathcal{S S}}(t)=T_{\mathcal{S} \mathcal{S}}^{2} \mathcal{P}_{\mathcal{R} \mathcal{R}}\left(t_{*}\right)
\end{aligned}
$$


lead to evolving spectral indices

$$
\begin{aligned}
n_{\mathcal{R} \mathcal{R}}(t) & =n_{\mathcal{R R}}\left(t_{*}\right)-\left(A_{*}+B_{*} T_{\mathcal{R S}}\right) \frac{2 T_{\mathcal{R S}}}{1+T_{\mathcal{R S}}^{2}} \\
n_{\mathcal{R S}}(t) & =n_{\mathcal{R} \mathcal{R}}\left(t_{*}\right)-\frac{A_{*}}{T_{\mathcal{R S}}}-2 B_{*} \\
n_{\mathcal{S S}}(t) & =n_{\mathcal{R} \mathcal{R}}\left(t_{*}\right)-2 B_{*},
\end{aligned}
$$

where the $*$ on $A, B$ indicates evaluation at horizon crossing. These indices can alternatively be expressed in terms of the correlation fraction $\gamma_{\text {corr }}=\mathcal{P}_{\mathcal{R S}} / \sqrt{\mathcal{P}_{\mathcal{R} \mathcal{R}} \mathcal{P}_{\mathcal{S}}}=T_{\mathcal{R S}} / \sqrt{1+T_{\mathcal{R S}}^{2}}$ for which constraints have been determined by the PLANCK collaboration. The results of eq. (5.27) complete the specification of the evolution of these spectral indices for general modular inflation.

As mentioned earlier, the gravitational tensor power spectrum can be quantified in a variety of ways when isocurvature perturbations are present. Using the tensor-to-adiabatic scalar power ratio (3.24) leads via the evolution of $\mathcal{P}_{\mathcal{R} \mathcal{R}}$ to the evolution of $r$ as

$$
r=\frac{r_{*}}{1+T_{\mathcal{R S}}^{2}},
$$

i.e. to a suppression of $r$ for post-horizon crossing times, hence changing the relation between $r$ and $n_{T}$. Alternatively, this can again be expressed in terms of the correlation fraction $\gamma_{\text {corr }}$. For more than two fields the adiabatic dynamics (3.10) shows that the isocurvature correlators $\left\langle S^{I J}(\vec{k}) S^{K L}\left(\vec{k}^{\prime}\right)\right\rangle$ lead to a further suppression of the tensor ratio, turning the above relation again into an inequality, a fact that was anticipated in [13] on the basis of a two-field discussion.

\section{$7 \quad j$-inflation}

There are a number of prominent modular forms that can be used within the framework of modular inflation. The model considered in [8] is based on a function that is basic to all modular functions, the $j$-function, which up to scaling is the Klein invariant $J(\tau)$. The fundamental nature of this function is indicated by its origin in the Eisenstein series $E_{4}$ and $E_{6}$, which provide a basis of the space of all modular forms of the full modular group $G(\mathbb{Z})=\mathrm{SL}(2, \mathbb{Z}) \subset \mathrm{SL}(2, \mathbb{R})$. Denoting the space of such modular forms by

$$
M_{*}(\mathrm{SL}(2, \mathbb{Z}))=\bigoplus_{w} M_{w}(\mathrm{SL}(2, \mathbb{Z}))
$$

where $M_{w}(\mathrm{SL}(2, \mathbb{Z}))$ denotes the spaces of weight $w$ forms, gives

$$
M_{*}(\mathrm{SL}(2, \mathbb{Z}))=\left\langle E_{4}, E_{6}\right\rangle
$$

where the Eisenstein series $E_{w}$ are defined in (4.18).

Modular invariant functions with respect to subgroups $\Gamma_{N} \subset \mathrm{SL}(2, \mathbb{Z})$ can be constructed by considering quotients of modular forms of equal weight. For the full modular group the dimension of the space $M_{w}(\mathrm{SL}(2, \mathbb{Z}))$ for even $w$ is given by [47]

$$
\operatorname{dim} M_{w}(\mathrm{SL}(2, \mathbb{Z}))=\left\{\begin{array}{ll}
{\left[\frac{w}{12}\right]} & \text { for } w \equiv 2(\bmod 12) \\
1+\left[\frac{w}{12}\right] & \text { for } w \neq 2(\bmod 12)
\end{array}\right\} .
$$


This shows that for even $w<12$ these spaces are at most one-dimensional. At weight $w=12$ one encounters the first cusp form, given by the Ramanujan form, which can be written in terms of the Dedekind eta function

$$
\eta(\tau)=q^{1 / 24} \prod_{n=1}^{\infty}\left(1-q^{n}\right),
$$

which is closely related to the partition function, hence the harmonic oscillator, as

$$
\Delta(\tau)=\eta(\tau)^{24}=q \prod_{n=1}^{\infty}\left(1-q^{n}\right)^{24}
$$

or alternatively in terms of the Eisenstein series (4.18) as

$$
\Delta(\tau)=\frac{E_{4}^{3}(\tau)-E_{6}^{2}(\tau)}{1728} .
$$

This form does not vanish on $\mathcal{H}$, hence one can obtain modular functions without poles on $\mathcal{H}$ by using $\Delta$ as the denominator.

An important modular invariant function obtained in this way is the $j$-function

$$
j(\tau):=\frac{E_{4}^{3}(\tau)}{\Delta(\tau)} .
$$

Up to a factor $j(\tau)$ is the Klein invariant $J(\tau)=j(\tau) / 1728$ [52]. The different normalizations are motivated by the fact that the Fourier expansion of $j(\tau)$ has integral coefficients

$$
j(q)=\frac{1}{q}+744+196884 q+21493760 q^{2}+864299970 q^{3}+20245856256 q^{4}+\cdots
$$

while the Klein invariant has nice values at the parabolic and elliptic points of the fundamental domain of the modular group. Different definitions and normalizations of $\Delta$ exist in the literature, but all definitions of the $j$-functions are such that they lead to the same $q$-expansion. The valence formula (5.16) implies that the $j$-function is holomorphic on $\mathcal{H}$, with a simple pole at infinity and a triple zero at $\xi_{3}=e^{2 \pi i / 3}$. This follows from the fact that the Ramanujan form $\Delta$ is holomorphic on $\mathcal{H}$ with a simple zero at infinity, hence is non-vanishing on the upper halfplane. The Eisenstein series $E_{4}$ is homolorphic with a simple zero at $\xi_{3}=e^{2 \pi i / 3}$, hence is non-vanishing on $\mathcal{H}$ and at infinity.

The $j$-invariant is an ubiquitous function which plays an important role in completely different contexts. After having been discussed earlier by Kronecker in 1857 [53] in the context of complex multiplication, and Hermite in 1858 [54] in the context of solving the quintic equation, it was interpreted by Dedekind in 1877 [55] as the function that maps the fundamental domain one-to-one onto $\mathbb{C}$. The $j$-function arises also in the geometry of elliptic curves and in the representation theory of the Fischer-Griess monster group. The connection to the latter arises because its Fourier coefficients encode the dimensions of the representations of this finite group, thereby linking $j$ to the largest of the finite simple groups via the theory of vertex algebras [56]. Part of the importance of the $j$-function 


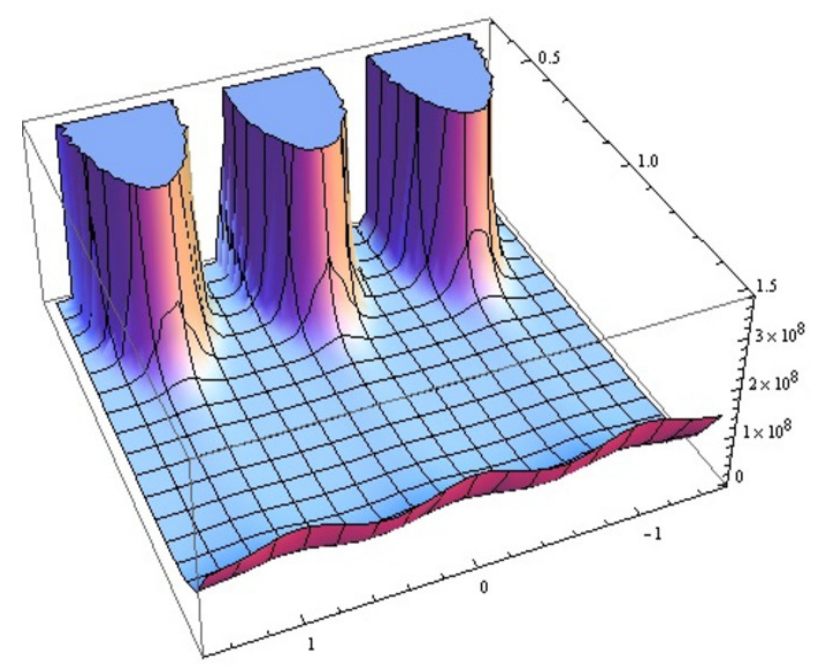

Figure 1. The $j$-function close to the boundary $(\operatorname{Im} \tau)=0$ in a region of the upper halfplane $\mathcal{H}$ centered around $(\operatorname{Re} \tau)=0$.

derives from the fact that every modular function on the upper halfplane can be expressed as a rational function of $j$ [47]. A brief history of this tantalizing object can be found in [57].

The Ramanujan form does not appear to be less versatile, with applications that range from a geometric interpretation as a motivic modular form, to partition functions of the bosonic string, as well as the entropy of certain types of black holes.

Inflationary potentials can be constructed by considering dimensionless functions $F(j, \bar{j})$. An immediate class of examples that can be considered is given by

$$
V_{p}\left(\phi^{1}, \phi^{2}\right)=\Lambda^{4}|j(\tau)|^{2 p}
$$

More general classes of models that could be considered are based on potentials of the form

$$
V_{p, q}\left(\phi^{1}, \phi^{2}\right)=\Lambda^{4}\left|j_{q}(\tau)\right|^{2 p}
$$

where $j_{q}(\tau)$ for $q$ prime are modular functions at level $q$ derived from $j$.

In the remainder of this paper the focus will be on the model with potential

$$
V\left(\phi^{1}, \phi^{2}\right)=\Lambda^{4}|j(\phi)|^{2} .
$$

Figure 1 shows a graph of the absolute value of the $j$-function close to the boundary $(\operatorname{Im} \tau)=0$ of a domain $X$ that is centered around $(\operatorname{Re} \tau)=0$.

\section{Observables of $j$-inflation}

The satellite probes WMAP [36] and PLANCK [37, 38] have constrained the scalar power spectrum, in particular its amplitude and spectral index, and put bounds on the tensor amplitude. These results thus provide a set of observables $\left(A_{\mathcal{R} \mathcal{R}}, n_{\mathcal{R} \mathcal{R}}, r\right)$ for early universe models. 


\subsection{Modular form of observables}

In order to express the observables in terms of the basic functions of $j$-inflation, it is useful to write the slow-roll parameters $\epsilon_{I}$ in terms of the Eisenstein series. The construction of the derivatives outlined above leads in the special case of the Eisenstein series to

$$
\begin{aligned}
& E_{4}^{\prime}=\frac{2 \pi i}{3}\left(E_{4} E_{2}-E_{6}\right) \\
& E_{6}^{\prime}=\pi i\left(E_{6} E_{2}-E_{4}^{2}\right),
\end{aligned}
$$

relations that were originally obtained by Ramanujan [58]. The appearance of the quasimodular Eisenstein series $E_{2}$ changes the transformation behavior of derivatives of modular forms.

The observables of $j$-inflation can then be obtained by using in addition the derivatives of the $j$-functions, which can be obtained as

$$
\begin{aligned}
\frac{j^{\prime}}{j} & =-2 \pi i \frac{E_{6}}{E_{4}} \\
\frac{j^{\prime \prime}}{j} & =-\frac{2 \pi^{2}}{3}\left(4 \frac{E_{6}^{2}}{E_{4}^{2}}+3 E_{4}-\frac{E_{6} E_{2}}{E_{4}}\right) .
\end{aligned}
$$

This leads to the Eisenstein form of the slow-roll parameters

$$
\epsilon_{I}=-2 \pi i \frac{M_{\mathrm{Pl}}}{\mu}\left(\frac{E_{6}}{E_{4}}+(-1)^{I} \frac{\bar{E}_{6}}{\bar{E}_{4}}\right),
$$

and the parameter that determines the slow-roll acceleration of the scale parameter $a(t)$ is given by

$$
\epsilon_{V}=\frac{1}{2} G^{I J} \epsilon_{I} \epsilon_{J}=8 \pi^{2}(\operatorname{Im} \tau)^{2} \frac{M_{\mathrm{Pl}}^{2}}{\mu^{2}}\left|\frac{E_{6}}{E_{4}}\right|^{2} .
$$

This implies that $\ddot{a}>0$ for values of $\tau$ that are close to the zero of the weight six Eisenstein series, which is given by $\tau=i$. At the point $\tau=i$ the Eisenstein series $E_{4}$ does not have a zero or a pole.

The spectral index expressed in terms of the Eisenstein series then takes the form

$$
\begin{aligned}
n_{\mathcal{R} \mathcal{R}}= & 1-\frac{8 \pi^{2}}{3}(\operatorname{Im} \tau)^{2} \frac{M_{\mathrm{Pl}}^{2}}{\mu^{2}}\left[8\left|\frac{E_{6}}{E_{4}}\right|^{2}-3 \operatorname{Re}\left(\frac{E_{6} \bar{E}_{4}^{2}}{E_{4} \bar{E}_{6}}\right)+\operatorname{Re}\left(\frac{E_{6}}{E_{4}} \bar{E}_{2}\right)\right] \\
& +8 \pi \frac{M_{\mathrm{Pl}}^{2}}{\mu^{2}}(\operatorname{Im} \tau) \operatorname{Re}\left(\frac{E_{6}}{E_{4}}\right),
\end{aligned}
$$

in dependence of $\tau=\phi / \mu$. The last term on the r.h.s. is induced by the curved target space metric. It combines with the $E_{2}$-term in the square bracket to an expression that contains the almost holomorphic modular form $\widehat{E}_{2}$ as a factor

$$
n_{\mathcal{R} \mathcal{R}}=1-\frac{8 \pi^{2}}{3}(\operatorname{Im} \tau)^{2} \frac{M_{\mathrm{Pl}}^{2}}{\mu^{2}}\left[8\left|\frac{E_{6}}{E_{4}}\right|^{2}-3 \operatorname{Re}\left(\frac{E_{4}^{2}}{E_{6}} \frac{\bar{E}_{6}}{\bar{E}_{4}}\right)+\operatorname{Re}\left(\widehat{E}_{2} \frac{\bar{E}_{6}}{\bar{E}_{4}}\right)\right]
$$


This can also be derived as a specialization of the general modular inflation result for $n_{\mathcal{R} \mathcal{R}}$ derived earlier in the paper.

The amplitude $A_{\mathcal{R}}$ of the scalar power spectrum can be expressed in terms of the $j$-function and the Eisenstein series $E_{w}$ as

$$
A_{\mathcal{R} \mathcal{R}}=\frac{1}{192 \pi^{4}}\left(\frac{\Lambda^{2} \mu}{M_{\mathrm{Pl}}^{3}}\right)^{2} \frac{1}{(\operatorname{Im} \tau)^{2}}\left|\frac{E_{4}}{E_{6}}\right|^{2}|j(\tau)|^{2}
$$

where the r.h.s. is to be evaluated with the inflaton values $\tau^{I}=\phi^{I} / \mu$ such that $n_{\mathcal{R} \mathcal{R}}$ and the tensor-to-scalar ratio $r$, considered below, are within the experimental range. Once $n_{\mathcal{R} \mathcal{R}}$ (and $r$ ) have been used to determine $\tau_{*}^{I}$ at the pivot scale one can use the experimental result for $A_{\mathcal{R} \mathcal{R}}$ to determine the energy scale $\Lambda$ of $j$-inflation. The isocurvature power at horizon crossing is the same as that of the adiabatic perturbation $\mathcal{P}_{\mathcal{S S}}=\mathcal{P}_{\mathcal{R} \mathcal{R}}$ and the cross correlation vanishes $\mathcal{P}_{\mathcal{R} S}=0$.

The tensor-to-scalar ratio $r$ of multifield inflation with curved targets (3.25) takes for modular inflation the form $r=8(\operatorname{Im} \tau)^{2} \delta^{I J} \epsilon_{I} \epsilon_{J}$, and with the $j$-inflation expression for the parameters $\epsilon_{I}$ one obtains the Eisenstein form for $r$ as

$$
r=128 \pi^{2}(\operatorname{Im} \tau)^{2} \frac{M_{\mathrm{Pl}}^{2}}{\mu^{2}}\left|\frac{E_{6}}{E_{4}}\right|^{2} .
$$

In the lowest order in the slow-roll approximation this determines the tensor spectral index via (3.28).

The evaluation of the observables is here at $t_{*}$, the time at which the pivot scale crosses the horizon during inflation. The first constraint on the parameters of the model can be obtained from the requirement that the number of e-folds

$$
N_{*}=\int_{t_{*}}^{t_{e}} d t H(t)
$$

should fall into the standard range $N_{*} \in[60,70]$. This interval is not sharp, and values within a wider range have been considered in the literature. The input for the computation of the number of e-folds is the inflationary dynamics which in the slow-roll approximation takes for $j$-inflation the form

$$
\dot{\phi}^{I}=\frac{2 \pi i^{I}}{\sqrt{3}} \frac{1}{G}\left(\frac{M_{\mathrm{Pl}}}{\mu}\right) \Lambda^{2}\left(\frac{E_{6}}{E_{4}}+(-1)^{I} \frac{\bar{E}_{6}}{\bar{E}_{4}}\right)|j|,
$$

where $G=\left(\mu / \phi^{2}\right)^{2}$ is the conformal factor of the modular inflation target space metric. Integrating the $j$-function then leads to the number of e-folds

$$
N_{*}=\frac{1}{\sqrt{3}} \frac{\Lambda^{2}}{M_{\mathrm{Pl}}} \int_{t_{*}}^{t_{e}} d t|j(\tau)| .
$$

\section{2 $j$-inflation observables and the PLANCK probe}

The parameter space of $j$-inflation is stratified by the energy scale $\mu$ that enters all the observables. Given a specific choice of $\mu$ and the pivot value $\phi_{*}^{I}$ of the inflaton, the spectral 


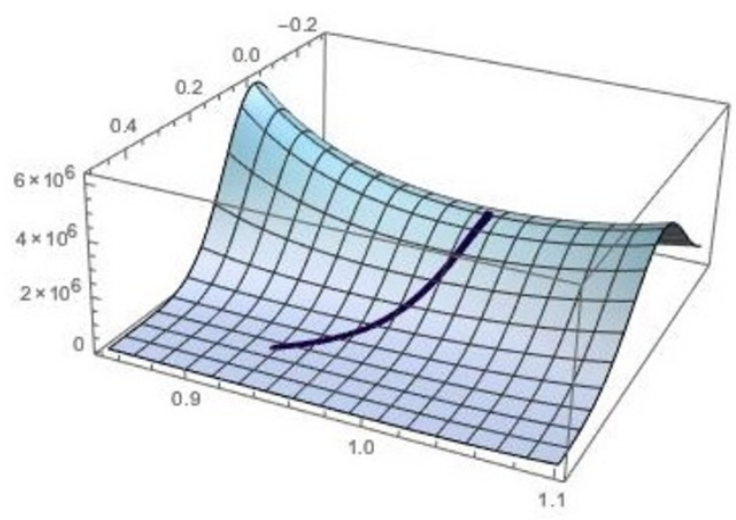

Figure 2. A close-up of the $j$-inflation potential in the neighborhood of $\tau=i$. The curved path indicates an $N_{*}=60$ trajectory.

indices $n_{\mathcal{R} \mathcal{R}}$ and $n_{T}$, as well as the tensor ratio $r$, can be computed from the Eisenstein formulae above. Furthermore, the scale $\Lambda$ can be obtained via eq. (8.7) from the PlancK scalar amplitude [37, 38], which in turn allows to determine the number of e-folds $N_{*}$.

A detailed scan of the parameter space given by $\left(\mu, \tau_{*}\right)$ can be performed, leading to different neighborhoods $U_{\mu}\left(\tau_{*}\right)$ in the upper halfplane that can be tested against the satellite probe constraints. The form of the slow-roll parameters $\epsilon_{I}$ in eq. (8.3) and the scalar spectral index in eq. (8.5) show that the horizon crossing value of the inflaton should be chosen in a neighborhood of the zero of the Eisenstein series $E_{6}$, which can be obtained from the valence formula (5.16) as $\tau=i=\sqrt{-1}$. The Eisenstein series $E_{2}$ and $E_{4}$ are finite at this point [47]

$$
E_{2}(i)=\frac{3}{\pi}, \quad E_{4}(i)=\frac{3 \Gamma(1 / 4)^{8}}{(2 \pi)^{6}}
$$

hence $\epsilon_{I}, n_{\mathcal{R} \mathcal{R}}$ and $r$ are regular functions in this neighborhood. A zoom of the potential close to this point with a particular inflaton trajectory is shown in figure 2. It illustrates in more detail the ridge along the $(\operatorname{Re} \tau)=0$ line that is suppressed in the large scale view of the potential of figure 1 .

After fixing the scale $\mu$ at a super-Planckian value, the inflaton values at horizon crossing can be chosen such that after integrating the $j$-inflation dynamics (8.10) the orbit $\phi^{I}(t)$ leads to a number $N_{*}$ of e-folds between horizon crossing and the end of inflation that falls within the standard range $N_{*}=[50,70]$. The field $\left(\phi^{I}\right)$ therefore traverses a super-Planckian distance in field space during inflation. Figure 3 gives an illustration of the behavior for a few trajectories in the target space $X$ associated to different scales $\mu$ that lead to the central value $N_{*}=60$ and are consistent with CMB phenomenology.

These models in particular all lead to spectral indices $n_{\mathcal{R} \mathcal{R}}=0.96 *$, compatible with the PLANCK result [37], and the tensor-to-scalar ratio takes values in the range $r \in\left[10^{-8}, 0.08\right]$, compatible with the result of the PLANCK Collaboration [59], which reports for the tensor ratio $r$ at the pivot scale $k_{p}=0.002 \mathrm{Mpc}$ the bound $r_{0.002} \leq 0.11$, while the BICEP2/Keck/PLANCK Collaboration reports $r_{0.05} \leq 0.12$ [60]. The energy scale $\Lambda$ 


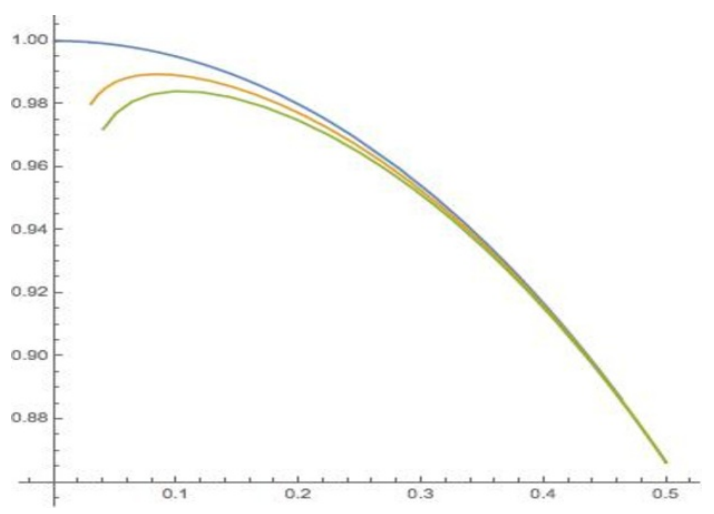

Figure 3. Trajectories of the inflaton for different initial conditions with e-fold numbers $N_{*}$ in the interval $[50,70]$.

determining the height of the potential includes the range $\Lambda \in\left[10^{-6}, 10^{-4}\right] M_{\mathrm{Pl}}$ for the realizations discussed here. More generally, the regions $U_{\mu}(\tau=i)$ around the slow-roll point $\tau=i$, identified above via the Eisenstein series, contain for varying $\mu$ many orbits that are consistent with PLANCK probe results. Moving too far from the slow-roll point violates the slow-roll condition, hence leads to tensor ratios $r$ that are too large, as expected. On the inflationary time scales during which large scale perturbations cross the horizon the effect on the observables of the transfer function $T_{\mathcal{O O}^{\prime}}$, which can be expressed in terms of the Eisenstein series $E_{w}$ using the results above and earlier in the paper, is small.

\section{Conclusion}

Modular inflation is a class of two-field models obtained as a specialization of multifield automorphic field theories by restricting the automorphic group $G(\mathbb{Z})$ to be given by the modular group $\mathrm{SL}(2, \mathbb{Z})$, or subgroups thereof. The framework considered here differs from inflation theories based on moduli, sometimes also called modular inflation. Moduli inflation posits that some of the moduli that arise in string theory, in particular in Calabi-Yau compactifications, are involved in the inflationary process. Cases of string theory induced potentials exhibiting automorphic symmetries provide special examples that fit into the more general framework of automorphic and modular inflation considered here and in $[8,9]$.

Modular inflation models present the simplest class of theories that allow to embed the shift symmetry into a group, in the process leading to a stratified theory space, in which the individual leaves that provide the building blocks of the resulting foliation are characterized by the weights and levels of the defining modular forms. The field theory space of automorphic inflation in general, and modular inflation in particular, has a nontrivial geometry that is encoded in the Riemannian metric $G_{I J}$ derived in a canonical way from the underlying group structure. In this paper a detailed description has been given of the general multifield curved target space dynamics and its specializations to two-field inflation and modular inflation, including a formulation of some of the variables that enter the phenomenological analysis for general modular potentials. An important problem arises from the fact that derivatives of modular forms are not modular, raising the issue of the 
modular nature of physical observables in modular invariant inflation. It was shown that the nonmodular contributions of the derivatives of the inflaton potential combine with the nonmodular terms induced by the curved target space into almost holomorphic modular forms that in turn lead to CMB observables that are almost holomorphic modular invariant.

An example of a modular function is given by the $j$-function, defined as a quotient of modular forms of weight twelve forms relative to the full modular group, i.e. level one. The inflationary two-field model that results from the simplest potential based on this function leads to a slow-roll phenomenology that is consistent with the observational results from the PlANCK satellite probe. The realization of $j$-inflation is constrained in particular by the bounds that have been established in the past decade first by WMAP and more recently by PLANCK and the joint PlANCK/BICEP collaboration, in particular the bounds on the gravitational contribution to the power spectrum.

\section{Acknowledgments}

It is a pleasure to thank Monika Lynker for discussions. This work was supported by a Faculty Research Grant at Indiana University South Bend and benefited in the early stages from the hospitality of the Simons Institute for Geometry and Physics.

Open Access. This article is distributed under the terms of the Creative Commons Attribution License (CC-BY 4.0), which permits any use, distribution and reproduction in any medium, provided the original author(s) and source are credited.

\section{References}

[1] A.A. Starobinsky, Multicomponent de Sitter (inflationary) stages and the generation of perturbations, JETP Lett. 42 (1985) 152 [Pisma Zh. Eksp. Teor. Fiz. 42 (1985) 124] [INSPIRE].

[2] D.S. Salopek, J.R. Bond and J.M. Bardeen, Designing density fluctuation spectra in inflation, Phys. Rev. D 40 (1989) 1753 [InSPIRE].

[3] M. Sasaki and E.D. Stewart, A general analytic formula for the spectral index of the density perturbations produced during inflation, Prog. Theor. Phys. 95 (1996) 71 [astro-ph/9507001] [INSPIRE].

[4] T.T. Nakamura and E.D. Stewart, The spectrum of cosmological perturbations produced by a multicomponent inflaton to second order in the slow roll approximation, Phys. Lett. B 381 (1996) 413 [astro-ph/9604103] [INSPIRE].

[5] S. Groot Nibbelink and B.J.W. van Tent, Scalar perturbations during multiple field slow-roll inflation, Class. Quant. Grav. 19 (2002) 613 [hep-ph/0107272] [INSPIRE].

[6] J.-O. Gong and E.D. Stewart, The power spectrum for a multicomponent inflaton to second order corrections in the slow roll expansion, Phys. Lett. B 538 (2002) 213 [astro-ph/0202098] [INSPIRE].

[7] F. Quevedo, Is string phenomenology an oxymoron?, arXiv:1612.01569 [INSPIRE].

[8] R. Schimmrigk, Automorphic inflation, Phys. Lett. B 748 (2015) 376 [arXiv:1412.8537] [INSPIRE]. 
[9] R. Schimmrigk, A general framework of automorphic inflation, JHEP 05 (2016) 140 [arXiv: 1512.09082] [INSPIRE].

[10] C. Gordon, D. Wands, B.A. Bassett and R. Maartens, Adiabatic and entropy perturbations from inflation, Phys. Rev. D 63 (2001) 023506 [astro-ph/0009131] [INSPIRE].

[11] C.M. Peterson and M. Tegmark, Testing multifield inflation: a geometric approach, Phys. Rev. D 87 (2013) 103507 [arXiv:1111.0927] [INSPIRE].

[12] J. Elliston, D. Seery and R. Tavakol, The inflationary bispectrum with curved field-space, JCAP 11 (2012) 060 [arXiv:1208.6011] [InSPIRE].

[13] D. Wands, N. Bartolo, S. Matarrese and A. Riotto, An observational test of two-field inflation, Phys. Rev. D 66 (2002) 043520 [astro-ph/0205253] [INSPIRE].

[14] B.A. Bassett, S. Tsujikawa and D. Wands, Inflation dynamics and reheating, Rev. Mod. Phys. 78 (2006) 537 [astro-ph/0507632] [INSPIRE].

[15] D. Wands, Multiple field inflation, Lect. Notes Phys. 738 (2008) 275 [astro-ph/0702187] [INSPIRE].

[16] S. Weinberg, Cosmology, Cambridge University Press, Cambridge U.K., (2008) [InSPIRE].

[17] D. Langlois and S. Renaux-Petel, Perturbations in generalized multi-field inflation, JCAP 04 (2008) 017 [arXiv: 0801.1085] [INSPIRE].

[18] D. Langlois, S. Renaux-Petel, D.A. Steer and T. Tanaka, Primordial perturbations and non-Gaussianities in DBI and general multi-field inflation, Phys. Rev. D 78 (2008) 063523 [arXiv:0806.0336] [INSPIRE].

[19] P.M. Saffin, The covariance of multi-field perturbations, pseudo-SUSY and $f_{\mathrm{NL}}$, JCAP 09 (2012) 002 [arXiv:1203.0397] [INSPIRE].

[20] L. McAllister, S. Renaux-Petel and G. Xu, A statistical approach to multifield inflation: many-field perturbations beyond slow roll, JCAP 10 (2012) 046 [arXiv:1207.0317] [INSPIRE].

[21] C.P. Burgess, M.W. Horbatsch and S. Patil, Inflating in a trough: single-field effective theory from multiple-field curved valleys, JHEP 01 (2013) 133 [arXiv:1209.5701] [INSPIRE].

[22] D.I. Kaiser, E.A. Mazenc and E.I. Sfakianakis, Primordial bispectrum from multifield inflation with nonminimal couplings, Phys. Rev. D 87 (2013) 064004 [arXiv:1210.7487] [INSPIRE].

[23] K. Schutz, E.I. Sfakianakis and D.I. Kaiser, Multifield inflation after Planck: isocurvature modes from nonminimal couplings, Phys. Rev. D 89 (2014) 064044 [arXiv:1310.8285] [INSPIRE].

[24] J. Ellis, M.A.G. García, D.V. Nanopoulos and K.A. Olive, Two-field analysis of no-scale supergravity inflation, JCAP 01 (2015) 010 [arXiv:1409.8197] [INSPIRE].

[25] M.A. Amin, M.P. Hertzberg, D.I. Kaiser and J. Karouby, Nonperturbative dynamics of reheating after inflation: a review, Int. J. Mod. Phys. D 24 (2014) 1530003 [arXiv:1410.3808] [INSPIRE].

[26] J.-O. Gong, Multi-field inflation and cosmological perturbations, Int. J. Mod. Phys. D 26 (2016) 1740003 [arXiv: 1606.06971] [inSPIRE]. 
[27] M. Dias, J. Frazer and D. Seery, Computing observables in curved multifield models of inflation - a guide (with code) to the transport method, JCAP 12 (2015) 030 [arXiv: 1502.03125] [INSPIRE].

[28] M.P. DeCross, D.I. Kaiser, A. Prabhu, C. Prescod-WEinstein and E.I. Sfakianakis, Preheating after multifield inflation with nonminimal couplings, I: covariant formalism and attractor behavior, arXiv:1510.08553 [INSPIRE].

[29] J.-O. Gong and T. Tanaka, A covariant approach to general field space metric in multi-field inflation, JCAP 03 (2011) 015 [Erratum ibid. 02 (2012) E01] [arXiv:1101.4809] [INSPIRE].

[30] D. Langlois, Lectures on inflation and cosmological perturbations, Lect. Notes Phys. 800 (2010) 1 [arXiv: 1001.5259] [INSPIRE].

[31] M.A. Amin, M.P. Hertzberg, D.I. Kaiser and J. Karouby, Nonperturbative dynamics of reheating after inflation: a review, Int. J. Mod. Phys. D 24 (2014) 1530003 [arXiv: 1410.3808] [INSPIRE].

[32] V.N. Lukash, Production of sound waves in the early universe, JETP Lett. 31 (1980) 596 [Zh. Eksp. Teor. Fiz. 31 (1980) 631].

[33] J.M. Bardeen, Gauge invariant cosmological perturbations, Phys. Rev. D 22 (1980) 1882 [INSPIRE].

[34] M. Sasaki, Large scale quantum fluctuations in the inflationary universe, Prog. Theor. Phys. 76 (1986) 1036 [INSPIRE].

[35] V.F. Mukhanov, Quantum theory of gauge-invariant cosmological perturbations, Sov. Phys. JETP 68 (1988) 1297 [Zh. Eksp. Teor. Fiz. 94 (1988) 1] [InSPIRE].

[36] WMAP collaboration, G. Hinshaw et al., Nine-year Wilkinson Microwave Anisotropy Probe (WMAP) observations: cosmological parameter results, Astrophys. J. Suppl. 208 (2013) 19 [arXiv: 1212.5226] [INSPIRE].

[37] Planck collaboration, P.A.R. Ade et al., Planck 2015 results XIII. Cosmological parameters, Astron. Astrophys. 594 (2016) A13 [arXiv: 1502.01589] [INSPIRE].

[38] Planck collaboration, P.A.R. Ade et al., Planck 2015 results XX. Constraints on inflation, Astron. Astrophys. 594 (2016) A20 [arXiv:1502.02114] [INSPIRE].

[39] A.A. Starobinsky, Spectrum of relict gravitational radiation and the early state of the universe, JETP Lett. 30 (1979) 682 [Pisma Zh. Eksp. Teor. Fiz. 30 (1979) 719] [INSPIRE].

[40] G. Shimura, Introduction to the arithmetic theory of modular forms, Princeton University Press, Princeton U.S.A., (1973).

[41] F. Diamond and J. Shurman, A first course in modular forms, Springer, New York U.S.A., (2005).

[42] F. Klein, Zur Theorie der Laméschen Funktionen (in German), Göttinger Nachrichten, Germany (1890), also LXIV in vol. 2 of Klein's Gesammelte Mathematische Werke.

[43] M. Galante, R. Kallosh, A. Linde and D. Roest, Unity of cosmological inflation attractors, Phys. Rev. Lett. 114 (2015) 141302 [arXiv: 1412.3797] [INSPIRE].

[44] B.J. Broy, M. Galante, D. Roest and A. Westphal, Pole inflation - shift symmetry and universal corrections, JHEP 12 (2015) 149 [arXiv:1507.02277] [INSPIRE]. 
[45] L. Euler, De summis serierum reciprocarum (in Latin), (1735), in Opera Omnia I-14, C. Boehm and G. Faber eds., (1925), pg. 73, translated by J. Bell, On the sums of series of reciprocals, [math.HO/0506415].

[46] T. Asai, M. Kaneko and H. Ninomiya, Zeros of certain modular functions and an application, Comm. Math. Univ. Sancti Pauli 46 (1997) 93.

[47] M. Koecher and A. Krieg, Elliptische Funktionen und Modulformen (in German), Springer, Germany, (1998).

[48] K. Ono, The web of modularity: arithmetic of the coefficients of modular forms and q-series, Amer. Math. Soc., U.S.A., (2004).

[49] D. Zagier, Elliptic modular forms and their applications, in The 1-2-3 of modular forms, Springer, Germany, (2008).

[50] G. Shimura, On a class of nearly holomorphic automorphic forms, Ann. Math. 123 (1986) 347.

[51] M. Kaneko and D. Zagier, A generalized Jacobi theta function and quasimodular forms, in The moduli space of curves, Birkhäuser, Boston U.S.A., (1995), pg. 165.

[52] F. Klein, Über die Transformationen der elliptischen Funktionen und die Auflösung der Gleichungen fünften Grades (in German), Math. Ann. 14 (1879) 111.

[53] L. Kronecker, Über die elliptische Funktionen für welche complexe Multiplication stattfindet (in German), Monatsberichte Königl. Preuss. Akad. Wiss. Berlin (1857) 455, in Werke, vol. 2, pg. 177 .

[54] C. Hermite, Sur la résolution de léquation du cinquième degré (in French), C. R. Acad. Sci. Paris Sér. I Math. (1858) 508.

[55] R. Dedekind, Ein Brief an Herrn Borchardts (in German), J. Angew. Reine Math. 83 (1877), also in Ges. Math. Werke I, pg. 174.

[56] R.E. Borcherds, Monstrous moonshine and monstrous Lie superalgebras, Invent. Math. 109 (1992) 405.

[57] J. Stillwell, Modular miracles, Amer. Math. Monthly 108 (2001) 70.

[58] S. Ramanujan, On certain arithmetical functions, Trans. Cambridge Philos. Soc. 22 (1916) 159.

[59] Planck collaboration, P.A.R. Ade et al., Planck 2013 results XVI. Cosmological parameters, Astron. Astrophys. 571 (2014) A16 [arXiv:1303.5076] [INSPIRE].

[60] BICEP2 and Planck collaborations, P.A.R. Ade et al., Joint analysis of BICEP2/Keck Array and Planck data, Phys. Rev. Lett. 114 (2015) 101301 [arXiv:1502.00612] [INSPIRE]. 